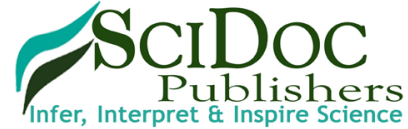

International Journal of Clinical \& Experimental Otolaryngology (IJCEO) ISSN 2572-732X

\title{
Surgical Treatment of the Frontal Sinus Lateral Mucocele with Multiple Sinus Bony Wall Defects
}

\author{
Case Report
}

Karina Jelagova ${ }^{1 *}$, Bogdanova $\mathrm{T}^{2}$

${ }^{1}$ Medical Department, Riga Stradins University, $6^{\text {th }}$ course, Riga, LV, Latvia.

${ }^{2}$ Dr. Med Jurshevich's Clinic of Aesthetic Medicine, Riga, Latvia.

\section{Abstract}

Paranasal sinus mucocele is a cystic formation filled with mucus, resulting from the obstruction of sinus ostium. The most common place of mucocele localization is frontal sinus, which is due to its specific anatomical architecture and a narrow passage of the frontal recess. Approximately in $70 \%$ mucocele localizes in a frontal sinus.

In this article we present the case report on 37 years old woman who applied to clinic of aesthetic medicine to get consultation about blepharoplasty possibilities. Patient complained on a mild bilateral upper eyelid ptosis, more significant on the left side. She also complained on recurrent nasal blockage, excessive nasal discharge, facial pain and pressure on her left side supraorbitally. Computered tomography (CT) for paranasal sinuses has been ordinated and lateral mucocele in a the left frontal sinus was diagnosed, as well as, inflammatory process in the left maxillary and ethmoidal sinuses.

Investigation and treatment options for this patient are discussed in this article.

Keywords: Frontal Sinus; Mucocele; FESS; Osteoplastic Flap; Frontal Sinus Obliteration.

\section{Introduction}

Paranasal sinus mucocele is a cystic formation filled with mucus, resulting from the obstruction of sinus ostium. The most common place of mucocele localization is frontal sinus, which is due to its specific anatomical architecture and a narrow passage of the frontal recess. Approximately in $70 \%$ mucocele localizes in a frontal sinus [1]. Mucus production in mucocele leads to its enlargement, which results in expansion of sinus cavity. Generally, affected by the pressure, sinus margins become thin and fragile, sometimes, resulting in bony defect and extension of the mucocele into the adjacent anatomical structures [2]. In such cases reconstructive craniofacial surgery has to be performed to prevent intracranial complications and face aesthetic deformities [3]. Real case of such a process is described below.

The aim of this article is to present a clinical case of 37 years old woman with frontal sinus lateral mucocele resulting in bone defects of anterior, posterior and inferior sinus walls.

\section{Case Report}

37 years old woman applied to the Dr. Med. E. Jurshevich's private clinic of aesthetic medicine with an intention to get a consultation of a plastic surgeon. Woman complained on a mild upper bilateral ptosis, which was more significant on the left side, therefore, initially the possibilities of upper blepharoplasty have been discussed. Although, she also mentioned that she has recurrent nasal blockage of the left side, breathing difficulties and excessive nasal discharge. Moreover, she also complained on facial pain and pressure on her left side, more expressive supraorbitally and above the topographical localization of the frontal sinus. Consequently, computered tomography (CT) investigation for paranasal sinuses has been ordinated. Analyzing the CT scan results, chronic rhinosinusitis of the left side was diagnosed: in maxillary and ethmoidal sinuses, unusual anatomy of frontal sinus with a total narrowing in a middle part and expansion of lateral part with a restricted mucous structure in it have been detected (Figure 1., Figure 2.). Moreover, three bone defects in the anterior wall of the frontal sinus were detected (Figure 3.).

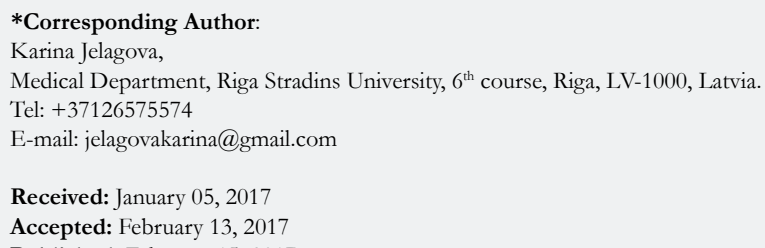

Copyright: Karina Jelagova ${ }^{\circ}$ 2017. This is an open-access article distributed under the terms of the Creative Commons Attribution License, which permits unrestricted use, distribution and reproduction in any medium, provided the original author and source are credited. 
Figure 1. Axial CT scan view. Inflammatory process in the left common frontal sinus and mucus opacification of its lateral part.

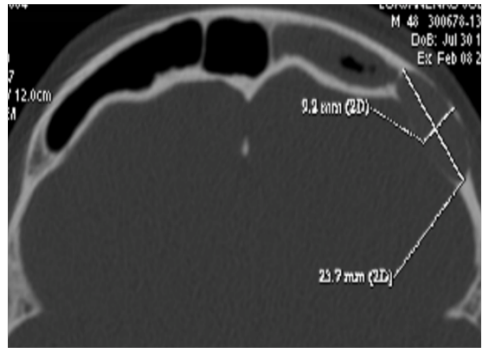

Figure 2. Coronal CT scan view. Inflammatory process in the left frontal, maxillary and ethmoidal sinuses.

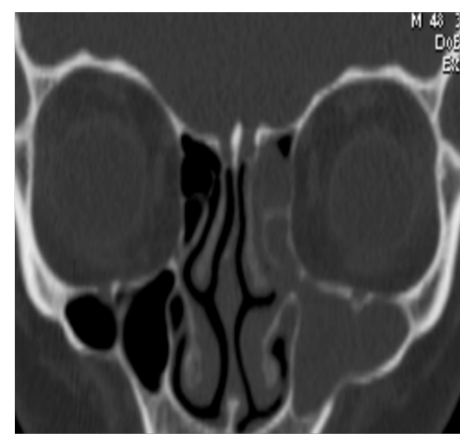

Figure 3. CT scan, reconstruction image. Three bone defects in the anterior wall of the frontal sinus.

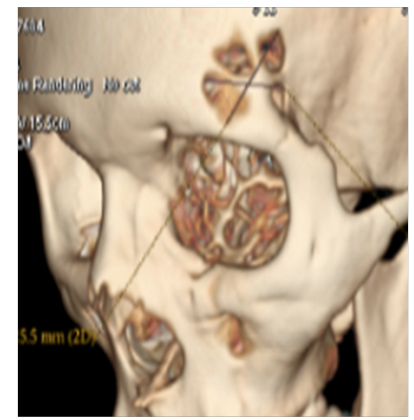

The floor of the frontal sinus also had bony defect, resulting in communication with orbital cavity (Figure 4.). According to the radiological findings, she was directed to receive a consultation of otorhinolaryngologist. Objectively, on endonasal endoscopic examination the patient had narrowed nasal passage with hyperemated and swelled mucosa in the left middle nasal chanal with purulent discharge. After conservative treatment with antibiotics, systemic decongestant and local andonasal steroids an alternate magnetic resonance imaging (MRI) scan has been done to define the localization and extension of the mucocele to other cavities. On T2W images hyperintense structure in a frontal sinus has been detected (Figure 5.). According to radiological findings, the patient had type Vamucocele, that means the erosion of both anterior and posterior walls of frontal sinus without or minimal intracranial extension. It has been decided to perform operation in two approaches: external for frontal sinus mucocele and endoscopic for maxillary sinus and ethmoidal cells.

Surgical process description. Bitemporal coronal incision was done, skin, aponeurotic tissue and pericranium were detached and elevated, preserving left supraorbital and left trochlear nerves. After this phase deffects in an anterior frontal wall were visualized, with mucocele mass protruding through it (Figure 6.). The next step was osteotomy performed with burr and anterior wall removal
(Figure 7.). After the sinus cavity opening the mucocele content was evacuated and all the mucosa lining of the frontal sinus was removed as well. The bone defect in the inferior frontal sinus wall, which communicated with orbita, was closed by flexible titanium micromash, as well, a narrow channel to common frontal sinus too (Figure 8.). Bone defect in posterior sinus wall was covered by fascia temporalis graft. To avoid relapse of mucocele, frontal sinus was obliterated by autologous fat material, taken from the paraumbilical region (Figure 9.). The bone cover was placed backwards and fixed with flexible titanium micromash and metal screws (Figure 10.). Skin and aponeurotic layers were lifted and a bitemporal coronal incision was closed with separate sutures and drainage was inserted into the postsurgical wound. In the following surgery stage functional endoscopic sinus surgery was performed. Using $0^{\circ}$ and $70^{\circ}$ telescopes the maxillotomy, anterior ethmoidotomy and frontotomy were done.

After the operation patient was prescribed three-day intravenous antibiotic course with Ceftriaxone $2 \mathrm{~g}$. once a day, Dexametazone $8 \mathrm{mg}$. once a day and Diclofenac sodium $75 \mathrm{mg}$. intramuscular injection twice a day. Three days after surgery patient was discharged from clinic for further treatment at home. In postclinical period she was prescribed one-week Cefadroxil 500 mg. orally twice a day, Diclofenacsodium $100 \mathrm{mg}$. orally once a 
Figure 4. Coronal CT scan. Bone defect in the floor of the frontal sinus.

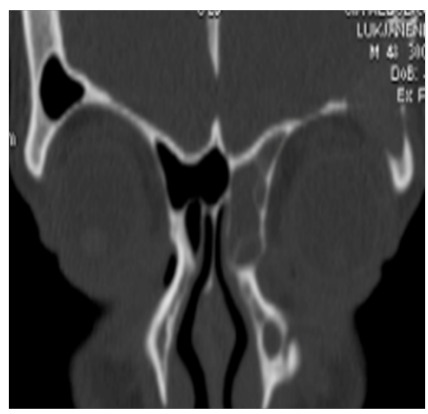

Figure 5. MRI scan, T2W. Hyperintense structure in a frontal sinus.

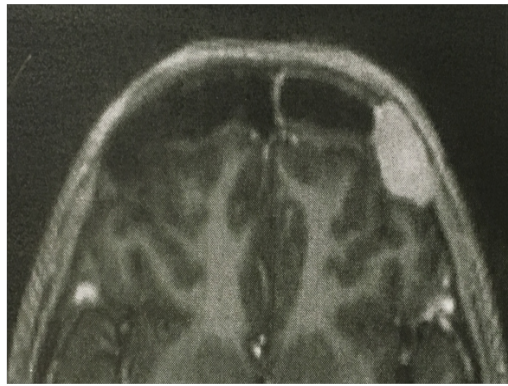

Figure 6. Bone defects in an anterior frontal sinus wall.

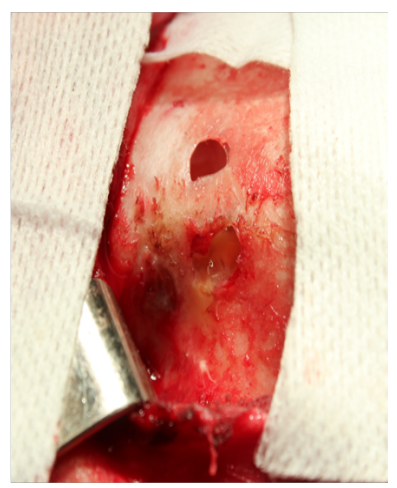

Figure 7. Removal of the anterior bony wall above mucocele.

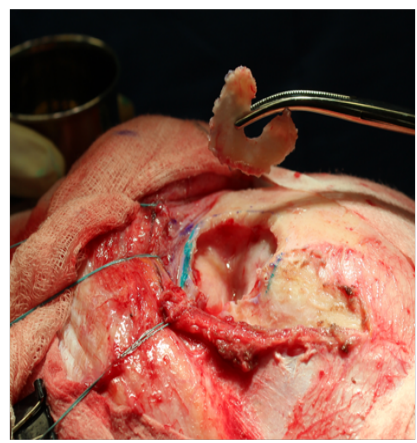

day and nasal irrigation with saline nasal spray. Later, after one week, patient was prescribed Mometazonefuroate.

On control otorhinolaringologist visit one week after surgery the patient had no complaints on any nasal symptoms, wound healed primarily. Further follow up was indicated.

On visit to otorhinolaryngologist six months after operation the patient did not have any complaints. Control computered tomography scan has been performed to visualize the result of the surgery. On CT scan obliterated frontal sinus without inflammation signs was visualized (Figure 11.). Inferior and anterior walls of the frontal cavity had no defects covered by micromash fixed by screws, as well as, the posterior wall of frontal sinus also had no defect (Figure 12.). No any inflammation signs have been detected in the left maxillary and ethmoidal sinuses after FESS (Figure 13). 
Figure 8. Bone defect in the inferior frontal sinus wall and channel to the common frontal sinus closure by flexible titanium micromash.

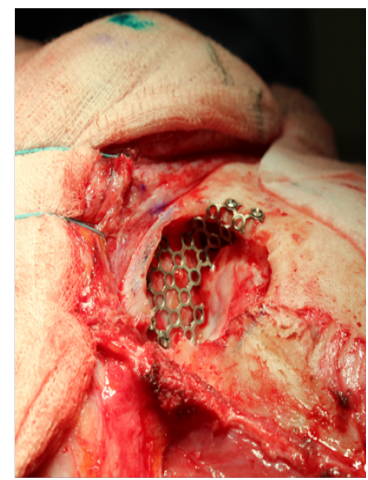

Figure 9. Frontal sinus obliteration by autologous fat material.

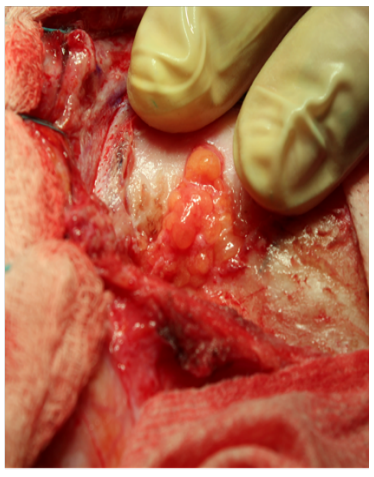

Figure 10. Bone cover placement backwards and fixation with flexible titanium micromash and metal screws.

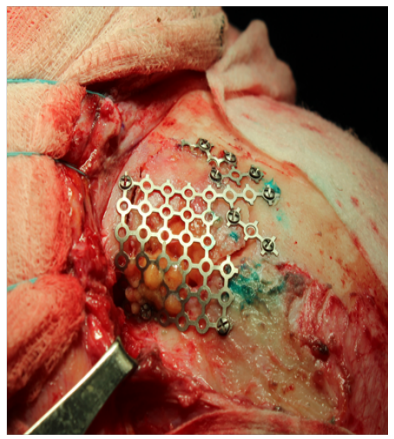

Figure 11. Axial CT scan. Obliterated frontal sinus without inflammation signs (6 months after surgery).

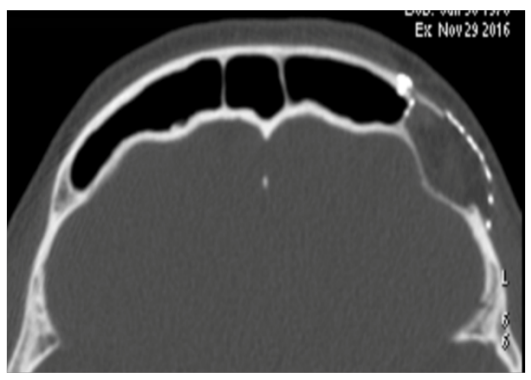

\section{Discussion}

Mucocele is mucus filled benign, slow-growing cystic formation, which develops due to obstruction of sinus ostium. Mucocele consists of fibrous capsule and a viscous content, secreted by goblet cells in the ciliated mucosa, which is, usually, sterile [1, 4]. Obstruction of sinus ostium most frequently occurs due to a mass lesion or secondary to fibrosis, inflam mation processes, osteoma, fibrous dysplasia, Paget disease, and rarely to malignancy, trauma with bony structure dislocation, or previous surgery and postoperative scar tissue and adhesions $[4,5]$. If any of trigger factors mentioned above occurs for continuous period of time the drainage pathway obstruction can lead to mucus retention and formation of mucocele. The most common localization of mucocele is frontal sinus due to its' specific anatomy of a narrow frontal recess. Blockage of the nasofrontal duct prevents the 
Figure 12. Sagittal CT scan. Inferior and anterior walls of frontal sinus covered by micromash. No defects in posterior wall (6 months after surgery).

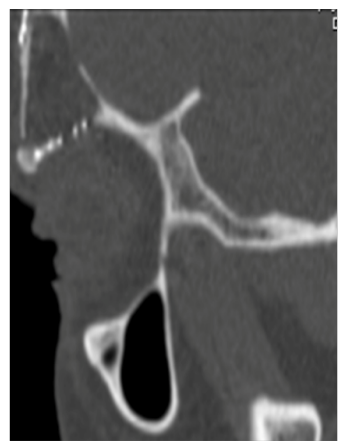

Figure 13. Coronal CT scan after FESS. Left maxillary and ethmoidal sinuses without signs of inflammation.

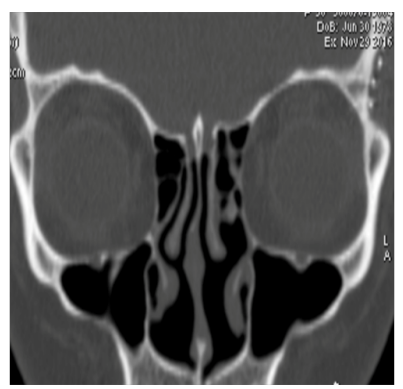

mucosal secretions from adequately draining and predisposes an obstructive epithelial cyst or mucocele to develop [5]. Growth of a mucocele can result in local bony destruction due to the local production of bone resorption factors such as prostaglandins, interleukin 1, collagenase and tumor necrosis factor and a direct effect of positive pressure within the mucocele $[6,7]$.

Clinical presentation of mucocele depends on its localization and involvement of adjacent anatomical structures. It is classified as rhinological, neurological, or ophthalmological symptoms $[8,9]$. Clinical presentation of mucocele is gradual, similar to chronic process. The most common symptoms of mucocele are rhinorrheae, nasal obstruction, facial pain or edema. If the mucocele transgresses the superior wall of orbital cavity than visual complaints like diplopia, diminution of vision, visual field defect, ptosis, orbital swelling, retro-orbital pain, displacement of eye-globe and proptosis are present [10]. In cases when mucocele enters fossa cranii anterior it can lead to confusion, meningitis, meningoencephalitis, pneumocephalus, brain abscess, seizures and cerebrospinal fluid leak [11].

The diagnosis of a paranasal sinus mucocele is a correlation between clinical, radiographic and pathologic findings. The most distinctive clinical signs are mentioned above. Computered tomography scan is considered to be the complementary method of choice in the investigation of mucoceles. On CT scan it is possible to evaluate the content of mucocele, sinus expansion, possible bone defects and extension of the mucocele mass to the adjacent structures. Typical view is isodense or mildly hyperdense sinus opacity in relation to the cerebral tissue, sinus margins expanded and usually thinned and peripheral calcification is sometimes seen. MRI scan is useful to identify the relation between mucocele, brain tissue, orbit and soft tissues [3,12]. The differences in MRI signal intensity appear to reflect variable water, protein and mucus ratio in the content of the obstructed sinus, which changes with time of evolution of the disease. Initially, the content is hyperhidrated, so the image is hypointense on T1weighted, and hyperintense on T2-weighted sequences. Over time, the protein contents increase, resulting in hyperintense images both on T1- and T2-weighted sequences. Moreover, it is possible to make the differential diagnosis with neoplasms: mucoceles have thin peripheral linear enhancement, when tumors have diffuse enhancement $[12,13]$. Radiologically, the most precise diagnostic result in difficult cases is possible when combining CT scan and MRI scan [14].

In described case, according to patients' anamnesis data, she has had chronic sinusitis for approximately 10 years. Continuous inflammation process, edema of nasal mucosa and specific anatomical predisposition could lead to obstruction of frontal recess, drainage disturbance and subsequent mucocele development. Bone destruction occurred due to prolonged pressure of mucocele mass on sinus walls. As mentioned previously in the text, the patient had defects in atrerior, posterior and inferior walls of the sinus. Defects in anterior wall were of a small diameter, therefore, no mucocele protrusion occurred through them. Concerning defects in inferior and posterior walls, usually, in such cases, intracranial or intraorbital complications develop. Moreover, if mucocele becomes infected osteitis, osteomyelitis, intracranial abscess, and meningitis may ensue [6]. In the patients' case correct symptom evaluation and diagnostic CT scan and MRI scan investigations were performed, so that, on time surgical treatment has been performed and development of several complications was avoided.

Fundamental treatment of mucocele is surgical. The goal of treatment is to widely open it to restore drainage and pneumatization of the sinus. Surgical treatment techniques of a mucocele include endoscopic, external, and combined approaches [7]. Surgical approaches are based on the size, location, and 
extent of the mucocele. Previously for treatment of frontal sinus mucocele external approach methods were used predominantly. Nowadays, traditional techniques of mucocele management consisting of frontal sinus obliteration have been supplanted with endoscopic drainage maneuvers $[6,15]$.

Currently, functional endoscopic sinus surgery (FESS) is considered the treatment of choice for paranasal sinuses mucoceles. The main objective of such method is the complete removal of both the lesion and the sinusal mucosa, thus preventing disease recurrence $[12,14]$. FESS is used for cases of mucocele with no extension to intraorbital or intracranial cavity and nor lateral onset of localization in frontal sinus. A classification system for endonasal frontal sinus surgery is based on the amount of the surgery. Draf type I-simple drainage in which anterior ethmoidal cells and processus uncinatus are removed. Draf type IIa-extended drainage in which the frontal sinus floor is resected from the lamina papyracea to the middle turbinate. Draf type IIb-extended drainage in which the frontal sinus floor is resected from the lamina papyracea to the nasal septum. Draf type III-endonasal median drainage in which the frontal sinus floor is resected from the right lamina papyracea to the left lamina papyracea with additional resection of the upper nasal septum and the inferior part of the interfrontal sinus septum. FESS is minimally invasive, preserves sinus architecture and leaves no facial scarring, which are notable advantages of this approach [14, 16]. Comparing FESS with external approach the recurrence rate is quite similar [15].

For complex cases with intracranial on intraorbital involvement, hypertrophic bone of recessus frontalis, difficult anatomy, lateral location of the frontal sinus mucocele, or suspectable malignancy, the use of external or combined approaches is considered. External approaches as Lynch-Howarth frontoethmoidectomy or osteoplastic flaps with sinus cavity obliteration may be used for mucocele management. Osteoplastic frontal sinus surgery with fat obliteration is very useful and successful in patients in whom frontal sinus cannot be treated effectively through an endonasal approach. In majority of cases the main aim or surgeon is to widen the drainage pathway of the sinus, but in cases with a high possibility of recurrence in junction with naturally narrow pathway obliteration is a choice to avoid recurrence. However, nowadays, use of external approaches should be carefully evaluated because of availability of modern minimally invasive FESS techniques [17, 18].

In particular case combined approach was performed: external for frontal sinus mucocele and FESS for maxillary sinus and ethmoidal cells. An external approach with osteoplastic flap and bitemporal coronal incision has been chosen, because of laterally localized mucocele, the bony defect in anterior, posterior and inferior frontal sinus walls, high risk of intracranial and intraorbital complication development and a good cosmetic result in comparison with Lynch-Hawarth approach, where the incision is made below the medial end of eyebrow. In this particular case cosmetic effect had significant importantce, because of the patients' young age. The obliteration of lateral part of the frontal sinus by autogenous fat material was performed due to its narrow junction with common frontal sinus and consequent increase of complication a recurrence risk.

\section{Conclusion}

Frontal sinus mucocele is gradually developing process with initially absent or nonspecific clinical performance. In majority of cases typical symptoms develop when mucocele reaches significant size, expands the sinus and extends to the sinus adjacent structures. Therefore, very careful evaluation of patients' anamnesis and symptom progression is important. Radiological investigations should be performed to precise the exact localization of mucocele and evaluate changes in structure of the sinus bone. Timely and precisely diagnosed mucocele allows to start treatment on its early stage and avoid the development of complications.

Surgical approach for mucocele management should be chosen according to its size, localization and involvement of adjacent anatomical structures. If the surgeon takes in consideration all aspects mentioned above, than it is possible to achieve the best treatment outcome results for patient.

\section{References}

[1]. Bruce M Wenig MD (2016) Atlas of Head and Neck Pathology. 3rd (Edn), Elsevier, Netherlands.

[2]. Thompson LDR, Wenig BM (2011) Diagnostic pathology: head and neck. 2nd (Edn) Elsevier, Netherlands.

[3]. A Prof Frank Gaillard (2010) Paranasal Sinus Mucocele. Radiopaedia Org.

[4]. Gregory G Capra, Peter N Carbone, David P Mullin (2012) Paranasal Sinus Mucocele. Head Neck Pathol. 6(3): 369-372.

[5]. Chan M Park, Enrico Stoffella, Jason Gile, Jeffrey Roberts, Alan S Herford, et al., (2012) Osteoplasty Flap Technique for Repair of Latent (30-Year) Post-Traumatic Frontal Sinus Mucocele: Case Report and Review of the Literature. J Oral Maxillofac Surg. 70(9): 2092-2096.

[6]. Paul W Flint, Bruce H Haughey, Valerie J Lund, John K Niparko, K Thomas Robbins, et al., (2015) Cummings Otolaryngology . 6th (Edn), Elsevier, Netherlands.

[7]. Weinzweig Jeffrey (2010) Plastic Surgery Secrets Plus. 2nd (Edition), Elsevier, Netherlands.

[8]. Fonseca Raymond J , Dexter Barber, Michael Powers, David E Frost (2013) Oral and Maxillofacial Trauma. (4th Edition), Elsevier, Netherlands.

[9]. Obeso S, Llorente JL, Rodrigo JP, Sanchez R, Mancebo G, et al., (2009) Paranasal sinuses mucoceles. Our experience in 72 patients. Acta Otorrinolaringol Esp. 60(5): 332-339.

[10]. Kim YS, Kim K, Lee JG, Yoon JH, Kim CH (2011) Paranasal sinus mucoceles with ophthalmologic manifestations: a 17 -year review of 96 cases. Am J Rhinol Allergy. 25(4): 272-275.

[11]. Beatriz Peral Cagigal, Javier Barrientos Lezcano, Raul Floriano Blanco, Jose Miguel Garcia Cantera, Luis Antonio Sanchez Cuellar, et al., (2006) Frontal sinus mucocele with intracranial and intraorbital extension. Neck and Facial Pathology. Med Oral Patol Oral Cir Bucal. 11: E527-30.

[12]. BrunaVilaça de Carvalho, James de Brito Correa, Izabella de campos Carvalho Lopes, Laura Filgueiras Mourao Ramos, Emilia Guerra pinto Coelho Motta, et al., (2013) Typical and atypical presentations of paranasal sinus mucocele at computed tomography. Radiol Bras. 46(6).

[13]. Pamela Van Tassel, Ya-Yen Lee, Bao-Shan Jing, Charles A De Pena (1989) Mucoceles of the Paranasal Sinuses: MR Imaging with CT Correlation. AJR Am J Roentqenol. 153(2): 407-12.

[14]. Sushil Kumar Aggarwal, Kranti Bhavana, Amit Keshri, Raj Kumar, Arun Srivastava (2012) Frontal sinus mucocele with orbital complications: Management by varied surgical approaches. Asian J Neurosurg. 7(3): 135-140.

[15]. Andy M Courson, James A Stankiewicz, Devyani Lal (2014) Contemporary Management of Frontal Sinus Mucoceles: A Meta-Analysis. Laryngoscope. 124(2): 378-86.

[16]. Mohammed K Al Komser, Andrew N Goldberg (2013) Unilateral transnasal endoscopic approach to frontal sinuses: Draf IIc. Allergy Rhinol. 4(2): e82-e87.

[17]. Stiernberg CM, Bailey BJ, Calhoun KH, Quinn FB (1986) Management of invasive frontoethmoidal sinus muco- celes. Arch Otolaryngol Head Neck Surg. 112(10): 1060-1063.

[18]. Voegels RL, Balbani AP, Santos Junior RC, O Butugan (1998) Frontoethmoidal mucocele with intracranial extension: A case report. Ear Nose Throat J. 77(2): 117-120. 